\title{
Influence of a reduced gravity on the volume fraction of a monolayer of spherical grains
}

\author{
S. Dorbolo, ${ }^{1,2}$ T. Scheller, ${ }^{1}$ F. Ludewig, ${ }^{1}$ G. Lumay, ${ }^{1,2}$ and N. Vandewalle ${ }^{1}$ \\ ${ }^{1}$ GRASP, Physics Department, University of Liège, B-4000 Liège, Belgium \\ ${ }^{2}$ F.R.S.-FNRS, Rue d'Egmont, 5, B-1000 Bruxelles, Belgium \\ (Received 4 May 2011; revised manuscript received 25 August 2011; published 19 October 2011)
}

\begin{abstract}
Centrifuge force is used to study granular materials in low gravity conditions. We consider a monolayer of noncohesive spherical grains placed on a plate. Reduced gravity conditions can be simulated in the plane by tilting or by rotating the plate. We compare both approaches experimentally. The volume fraction is found to increase with the apparent gravity and saturates. A model based on the exponential distribution of the Voronoi cell areas has been built and is in excellent agreement with the experimental data by extrapolating the fits of the data. Moreover, numerical simulations exhibit that more arches can be maintained at low apparent gravities than at high.
\end{abstract}

DOI: 10.1103/PhysRevE.84.041305

PACS number(s): 81.05.Rm, 45.70.Cc

\section{INTRODUCTION}

Most space mission scenarii require us to understand the low gravity behaviors of granular materials, since a major part of extraterrestrial soils belongs to this material family. This causes big concerns for any future human exploration of the moon and Mars. Indeed, granular matter already led to problems in past space exploration. On the lunar surface in December 1972, Apollo 17 astronauts needed to repair one of their lunar rover's fenders in an effort to keep the "rooster tails" of dust away from themselves and their gear.

Moreover, questions remain concerning the coherence of asteroids. These very small spatial objects are constituted by the agglomeration of dusts and small particles. A very nice review concerning the force acting for the cohesion of asteroids has been recently published [1]. In this paper, the authors analyzed very carefully the different contributions: van der Waals, friction, self-gravity, solar pressure, etc. These different ingredients play an important role in the determination of the shape and solidity of space objects. The internal structure is governed by the physics of a cohesive granular material. One of the main features is that the network of apparent contact is different from the network of forces, i.e., arches exist through the material. These arches support the weight of the pile while other grains do not play any role in the stability of the structure $[2,3]$.

A lot of ingredients have to be taken into account in the constitution of a granular assembly at low gravity. Basically, the present investigations aim to determine the influence of a reduced gravity on the volume fraction of an assembly of noncohesive beads. The considered system is simplified such that only the friction force can play a role in the granular medium. Cohesion can be neglected as the weight of the grains is large compared to van der Waals interactions (we use millimetric stainless steel beads). The restriction of the present methods is that they apply only on a monolayer of grains. On the other hand, this restriction allows us to have access to the position of all the grains.

A monolayer of grains was investigated recently [4-7]. Those works show that the normal force (beads on the plate) plays an important role as it governs the friction of the beads with the plate. In Refs. [4,5], the stability of the monolayer was studied when the plate is tilted by an angle $\alpha$. A critical angle $\alpha_{c}$ exists above which the monolayer collapses. This angle depends on the aspect ratio of the monolayer, on the monodispersity of the grains, on the bead-bead friction, and on the bead-plate friction. For a static monolayer, the bead-bead friction avoids the rolling motion. The monolayer can be considered as an assembly of blocks that does not slip down the plane because of the bead-plate friction. In Ref. [6], this block structure is destabilized by a slider that is allowed to roll down the plate because the beads can roll below the slider. More recently, Géminard et al. [8] studied the dynamics of a horizontal monolayer submitted to the motion of a conveyor belt. This remarkable experiment showed that the flow rate depends on the conveyor belt speed and not on the pressure. The role of force redirection toward the borders is questioned in a dynamical situation.

A lot of strategies exist to obtain zero-g conditions (parabolic flight, dropping tower, rocket, space station) or to obtain large gravity conditions (e.g., the Large Diameter Centrifuge at ESTEC). It is possible to simulate the conditions on Mars or the moon in parabolic flights. However, it is quite difficult to tune the gravity. Another way to decrease the influence of the gravity is to replace the air by a liquid which density can be varied [9]. On the other hand, the contact dynamics is deeply modified by such a method. More recently, the diamagnetism property of bismuth beads has been used to tune the apparent gravity [10]. In this paper, in order to test a gravity $g^{\star}$ lower than the Earth's gravity $g$, two experimental strategies have been approached (Fig. 1).

The first method consists in inclining the plane. The driving parameter is the angle $\alpha$ of the incline. The intensity of the simulated gravity $g^{\star}$ is given by $g \sin \alpha$. The problem with this configuration is that the normal force $\vec{N}$ depends also on the angle since its intensity is given by $m g \cos \alpha$, where $m$ is the mass of one bead. Consequently, the friction force $\vec{F}_{f}=\mu \vec{N}$, where $\mu$ is the static friction coefficient that depends also on the angle $\alpha$.

The second method consists in the rotation of the plate placed horizontally at a distance $R$ from the rotation axis. The generated gravity $g^{\star}$ is then given by $R \omega^{2}$, where $\omega(=2 \pi f)$ is the angular speed, $f$ being the frequency. In this case, the normal force remains the same regardless of the rotation speed. That is why most of the present work is devoted to the second method. The only restriction is that $g^{\star}$ is not uniform since the acceleration depends on $R$. In the 


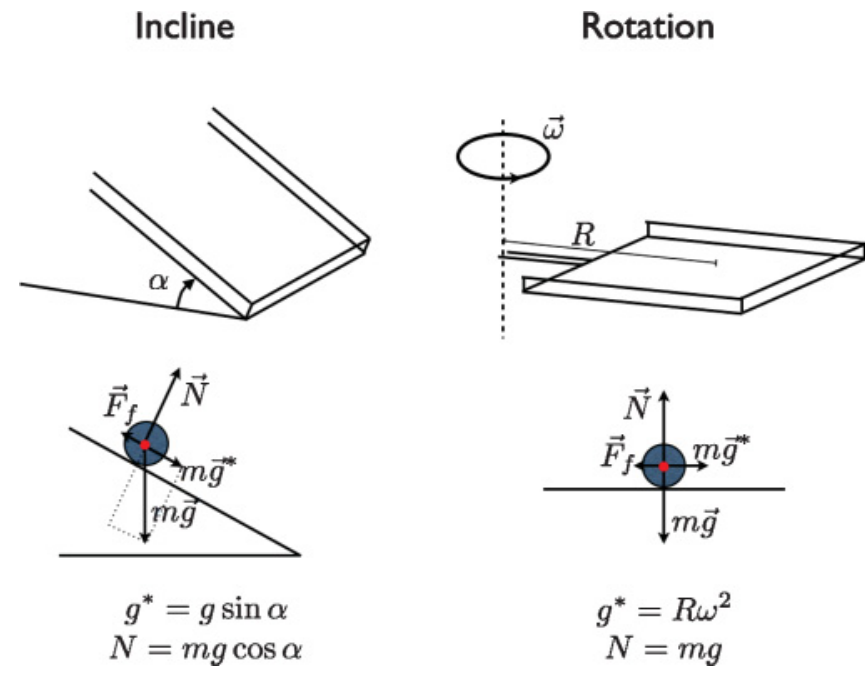

FIG. 1. (Color online) Generation of low apparent gravity. Left: The incline plane method consists in tilting a flat plate on which a monolayer of spherical grains is laid. The angle of incline drives the apparent gravity in the plane of the monolayer. Right: A flat plate is placed horizontally at the end of an arm that can rotate horizontally. The rotation generates a centrifuge force in the plane of the flat plate. Forces acting on one bead of the monolayer are represented at the bottom of the figure.

chosen geometry, the variations are about $5 \%$ of $g^{\star}$. Finally, for comparison purposes, the results obtained with the incline plane are also presented.

Numerical simulations have been performed. They are based on the nonsmooth dynamics of contacts [11,12]. This method allows us to access the force network. We found that the apparent gravity strongly influences the distribution of the force lines through the monolayer governing both geometry and volume fraction.

The rotating plate setup is described in the next section. The experimental method to determine the volume fraction is then explained in that section. The experimental results are shown and discussed in Sec. III. Numerical simulations are used to interpret the role of the apparent gravity on the monolayer system in Sec. IV. Conclusions are drawn in Sec. V.

\section{EXPERIMENTAL SETUP}

The plane was made of a glass plate with the dimensions $200 \times 300 \mathrm{~mm}^{2}$. On this plane, the spherical $(R=1 \mathrm{~mm}$ radius) stainless steel beads were placed. The plate was surrounded by a metallic border. The plate was then horizontally fixed at the extremity of an arm, which can rotate horizontally using an electrical motor. The middle of the plate was located at $R=1.6 \mathrm{~m}$ from the rotation axis. Figure 2 (top) shows a picture of the experimental setup. A battery was used to provide electrical power to the remote elements loaded on the centrifuge (LED lighting, the gate). The pictures were taken using a remote CANON EOS 400d camera (resolution $3888 \times 2592)$.

When rotating, due to centrifuge force, a grain placed on the plate moves away from the center of rotation, i.e., toward a side of the plate that we call the "bottom." At $10 \mathrm{~mm}$ before the bottom, we placed a gate. It consists in a metallic straight
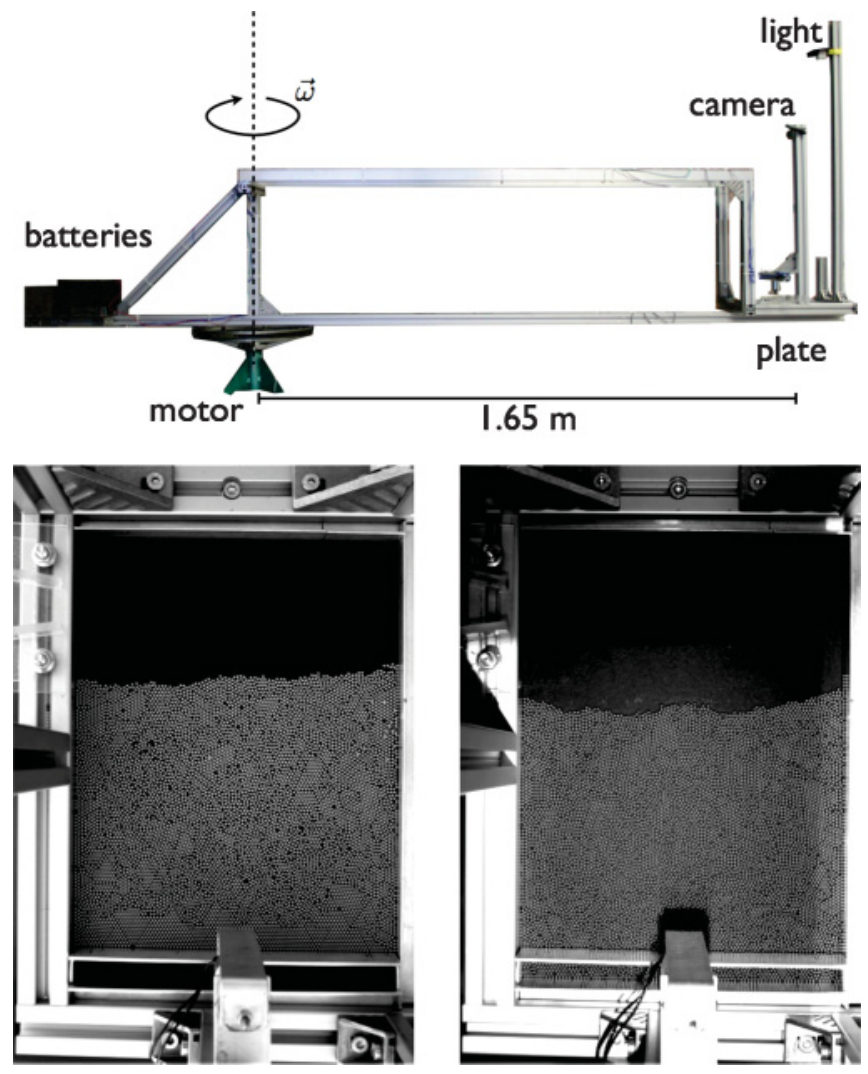

FIG. 2. (Color online) Top: Picture of the experimental setup of the rotating system. The plate is on the right of the picture. Bottom: Two snapshots of the monolayer taken from the top during a stabilized rotation speed. On the left, the gate is close. On the right, the monolayer is formed at the bottom of the plate after the aperture of the gate.

edge placed perpendicular to the arm in order to stop the grains from rolling to the bottom. Using a remote control, it is possible to open the gate when the system rotates. If the gate is placed further from the bottom, as the grains move toward the bottom, the Coriolis force plays an important role. One centimeter is a good compromise between the motion to allow a reorganization according to the considered $g^{\star}$ and a low speed condition to avoid the Coriolis force. Finally, a plexiglas shield was placed around the plate in order to drastically reduce the influence of the air.

The experimental procedure was as follows: The rotation speed was set first. After stabilization of the speed, the $N=$ 7600 grains were released by the gate. They move (roll and/or slide) to the bottom, i.e., decompaction occurs and the grains are reorganized at the bottom under the action of the centrifugal force. A picture is taken while the system is still rotating. The system is stopped and a new experiment can start. In Fig. 2 (bottom), two pictures present a typical experiment. On the left, the grains are blocked by the gate. On the right, the grains reorganize on the bottom border due to the apparent gravity $g^{\star}$. The monolayer reorganization behaves similarly to a vertical monolayer as ordered domains grow [13].

Some limitations should be noted. The apparent gravity $g^{\star}$ must be larger than $0.3 \mathrm{~m} / \mathrm{s}^{2}$ in order to allow the grains to roll. The apparent gravity is also limited at the large value 
$\left(g^{\star}<2 \mathrm{~m} / \mathrm{s}^{2}\right)$ to maintain monolayer conditions. The acceleration $g^{\star}$ is measured following $R \omega^{2}, R$ being the distance between the axis of rotation and the middle of the plate. As the plate measures $300 \mathrm{~mm}$ in height, there exists a gravity gradient of about $5 \%$.

The volume fraction (we should call it surface fraction) $\eta$ is measured using imaging. The beads are lighted by a LED lamp located at $500 \mathrm{~mm}$ above the plate. The beads are located by determining the spot reflection at the center of each bead. The surface $S$ occupied by the bead is measured. The volume fraction is obtained by dividing the surface of the beads $N \pi r^{2}$ by the measured area $S$.

For the incline, a similar method was used. The plane was tilted. The grains were then placed on the incline, the gate being shut. The gate was then open and a picture was taken after relaxation. The plane used was the same as the plane used in the centrifuge.

\section{EXPERIMENTAL RESULTS}

In Fig. 3, the volume fraction $\eta$ (red circles) obtained with the rotating system is plotted with respect to the reduced acceleration $g^{\star}$ given by $R \omega^{2}$. Five measurements by acceleration have been performed. A preliminary measurement that consists in the determination of the distribution of the values concludes to a Gaussian distribution. The averages and the standard deviations are then represented in Fig. 3 (blue squares).

Phenomenologically speaking, the following interpretation can be given. When the grains are released by the gate, they move toward the "bottom" of the plate. Their motions are stopped when grain-grain friction is larger than the centrifuge force or when they are geometrically blocked by the bottom or by other blocked beads. At low accelerations, the assembly is generated by low-speed grains and with a very low apparent

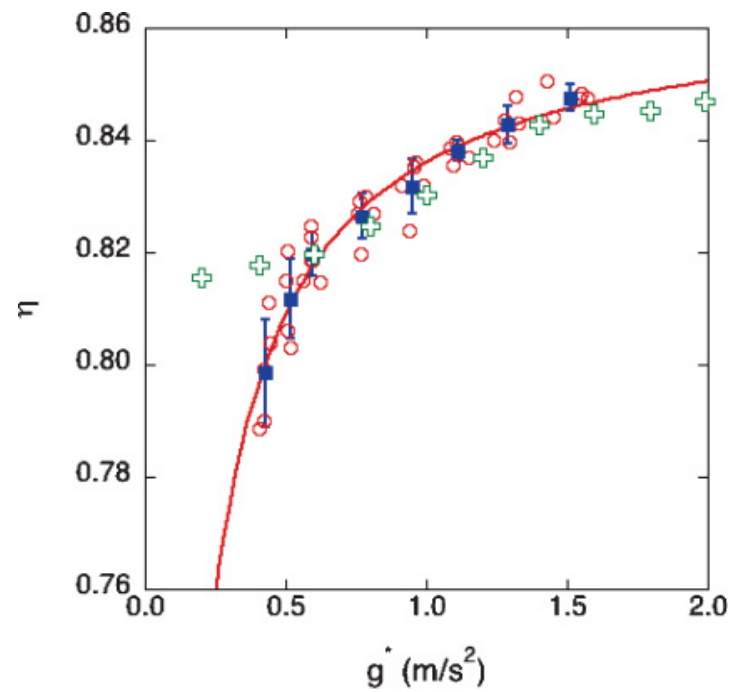

FIG. 3. (Color online) Influence of the reduced gravity $g^{\star}$ (centrifuge) on the volume fraction $\eta$ of a freshly made 2D packing of noncohesive spherical particles. The red circles correspond to the different experiments. The blue squares correspond to the mean value of the volume fraction for close values of $g^{\star}$. The continuous red line is a fit according to Eq. (4). Finally, the green crosses represent the volume fractions found by numerical simulations. weight (due to the in-plane apparent gravity). The assembly is rather loose, with arches existing through the heap. On the other hand, for large accelerations, the apparent weight of the grains is large; grain-grain friction cannot avoid grain motions. The volume fraction tends toward the characteristic value of the random close packing in two dimensions $(2 \mathrm{D}), \eta_{\mathrm{RCP}}^{2 \mathrm{D}} \approx 0.9$ $[14,15]$. When looking at the local geometry of the grains in a two-dimensional assembly of identical grains, a wide variety of configurations may be found if one considers the local packing fraction $\eta_{\ell}$ defined by the grain area $\pi r^{2}$ divided by the area of the Voronoi cell area associated with each grain [16]. This local measure should vary between the random loose packing value $\eta_{0}$ and the maximum packing fraction $\eta_{m} \approx 0.90$ corresponding to the regular hexagonal structure (crystallization).

The probability distribution of local densities has been addressed by earlier works in 3D [17,18] and in 2D [19]. In $2 \mathrm{D}$, the Voronoi cell area $S_{v}$ is very well modeled by a Gamma function [19]. The Voronoi tessellations have been performed for two typical experimental packings, namely at $g^{\star}=0.44$ and $1.45 \mathrm{~m} / \mathrm{s}^{2}$. The surface of each cell has been measured. Both typical cumulative distribution functions (CDF's), which represent the probability of finding a value larger than $S_{v}$, are presented in Fig. 4 in a semilog plot.

Two observations of the experimental CDF allow us to deduce a simplified distribution that takes into account the apparent gravity dependence:

(i) When the apparent gravity is increased, the CDF's are shifted toward lower Voronoi surfaces. Indeed, the surfaces are found to be larger than a given minimum surface $S_{m}$ that depends on the apparent gravity. The asymptotic value of $S_{m}$ for large $g^{\star}$ is given by the surface of the circumscribed hexagon to a bead, $S_{h}=2 \sqrt{3} R^{2}$.

(ii) At large apparent distribution, the Gamma distribution can be simplified to the form $\left(S_{v}-S_{m}\right) \exp \left[-\left(S_{v}-S_{m}\right) / S_{0}\right] d S_{v}$

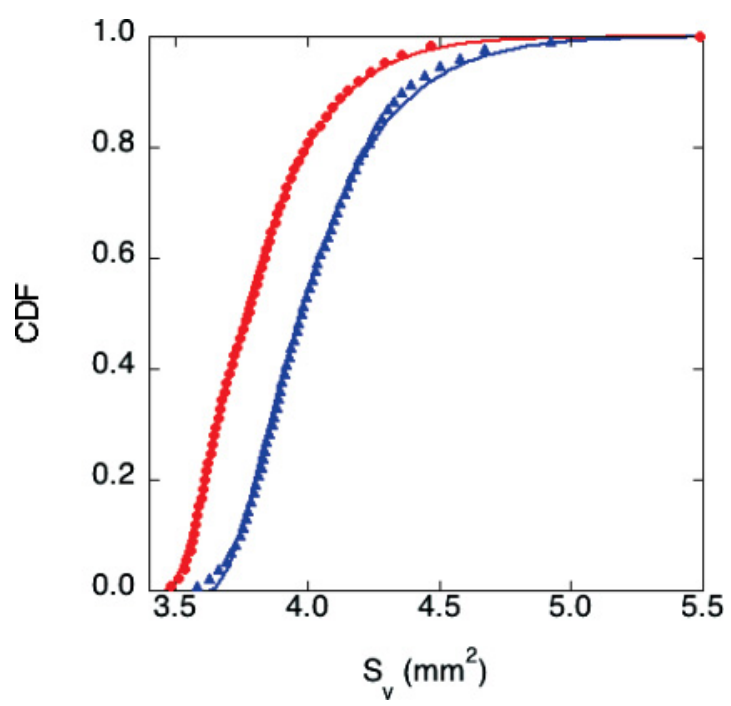

FIG. 4. (Color online) Experimental cumulative distribution function (CDF) of the Voronoi surface $S_{v}$ for two accelerations (centrifuge): $g^{\star}=0.44 \mathrm{~m} / \mathrm{s}^{2}$ (blue triangles) and $g^{\star}=1.45 \mathrm{~m} / \mathrm{s}^{2}$ (red circles). The continuous lines are fit with Eq. (3). Note that only $2 \%$ of the data are represented. 
where $\alpha$ is an exponent and $S_{0}$ is the characteristic Voronoi surface.

The function $S_{m}\left(g^{\star}\right)$ is to be convergent toward $S_{h}$ when $g^{\star}$ tends to infinity. On the other hand, the limit when $g^{\star}$ tends to zero cannot be considered as determined. Indeed, the packing fraction depends on the initial condition. Consequently, we choose to model the dependence of the minimum surface as

$$
S_{m}(g)=S_{h}\left(1+\gamma / g^{\star}\right),
$$

where $\gamma$ is the characteristic acceleration. Finally, the distribution function $P\left(S_{v}, g^{\star}\right)$ is given by

$$
P\left(S_{v}, g^{\star}\right) d S_{v}=\frac{1}{S_{0}}\left[S_{v}-S_{m}\left(g^{\star}\right)\right] \exp \left(-\frac{S_{v}-S_{m}\left(g^{\star}\right)}{S_{0}}\right)
$$

and the cumulative distribution function by

$$
\mathrm{CDF}=1-\exp \left(-\frac{S_{v}-S_{m}\left(g^{\star}\right)}{S_{0}}\right)\left(1+\frac{S_{v}-S_{m}\left(g^{\star}\right)}{S_{0}}\right) .
$$

The continuous lines in Fig. 4 represent the fit using Eq. (3). A good agreement with the experimental data is found with the simplified cumulative distribution.

Finally, the volume fraction $\eta\left(g^{\star}\right)$ can be calculated by dividing the surface of a bead $S_{b}$ by the average value $\left\langle S_{v}\left(g^{\star}\right)\right\rangle$ of a Voronoi cell given by $\int_{S_{m}}^{\infty} S_{v} P\left(S_{v}, g^{\star}\right) d S_{v}$. One finds

$$
\eta\left(g^{\star}\right)=\frac{S_{b}}{\left\langle S_{v}\right\rangle}=\frac{S_{b}}{2 S_{0}+S_{h}\left(1+\gamma / g^{\star}\right)} .
$$

This behavior is fitted on the data of Fig. 3 with only two free-fitting parameters, providing the values $S_{0}=0.08 \mathrm{~mm}^{2}$ and $\gamma=0.04 \mathrm{~m} / \mathrm{s}^{2}$ while $\eta(\infty)=0.86$ and $\eta(0)=0$. The agreement is excellent. The parameter $\gamma$ should reflect the acceleration for which a change in the geometrical structure of the pile or/and in the force network occurs. This range of acceleration is difficult to reach with the proposed experimental setup.

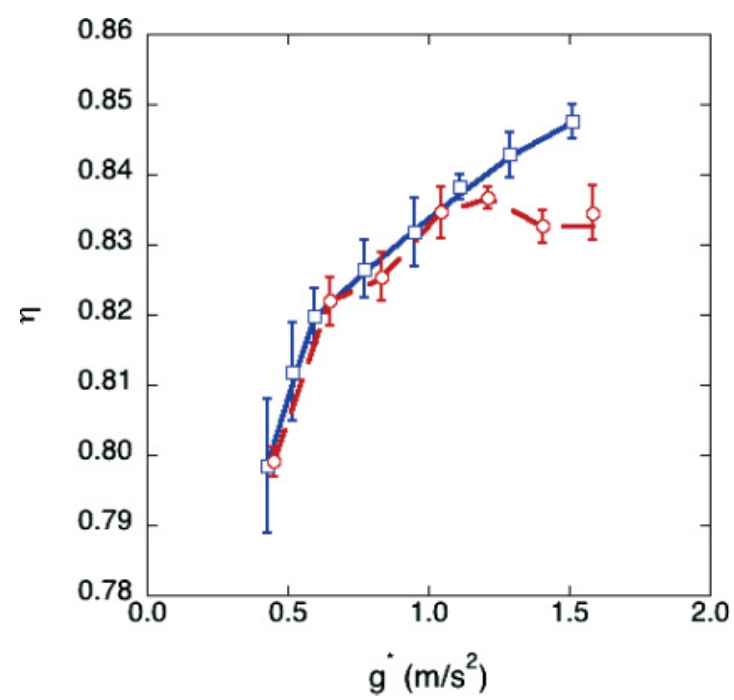

FIG. 5. (Color online) Comparison of the volume fraction $\eta$ vs the apparent gravity $g^{\star}$ obtained from the rotating system (blue squares) and the incline methods (red circles). The curves are guides to the eye.
A comparison between the incline plane and the rotating system is presented in Fig. 5. Blue squares and red circles correspond to the rotating plate and the incline plate, respectively. A good agreement is found between both experiments until $g^{\star}=1.2 \mathrm{~m} / \mathrm{s}^{2}$. Above this value, the volume fraction obtained with the incline plane is lower than for the rotating system. For large gravity, the normal force acting on the beads becomes lower and lower in the incline case. As soon as the gate is open, the grains not only roll, they slide in blocks. Consequently, the packing has no opportunity to reorganize according to the imposed apparent gravity and it maintains the low volume fraction character. The comparison really evidences the difference between both methods. The saturation of the volume fraction is higher in the rotating system. The large difference between the coefficient of friction for rolling and for sliding could explain the results.

\section{SIMULATIONS}

Numerical simulations based on nonsmooth dynamics of contacts (NSDC) [11,12] have been performed. The basis of these simulation methods is to consider both friction forces (normal and tangential) as a nonsmooth function of the speed. The simulation parameters include the bead-bead friction $\left(\mu_{\mathrm{b}-\mathrm{b}}=0.3\right)$, the bead-plate friction $\left(\mu_{\mathrm{b}-\mathrm{pl}}=0.3[20]\right)$, and the bead-bead coefficient of restitution $\varepsilon=0.9$. The experimental setup has been reproduced numerically: the plate measures $200 \mathrm{~mm}$ width, and 8000 spherical beads of $2 \mathrm{~mm}$ in diameter were released by a gate located $10 \mathrm{~mm}$ before the bottom. The volume fraction $\eta$ was computed with respect to the apparent gravity $g^{\star}$ and plotted in Fig. 3 (green crosses). The agreement is reasonable with the experimental data. A discrepancy is found for low values of the apparent gravity. After analysis, we found that the volume fraction depends more and more on the initial conditions when the apparent gravity is set to lower values.

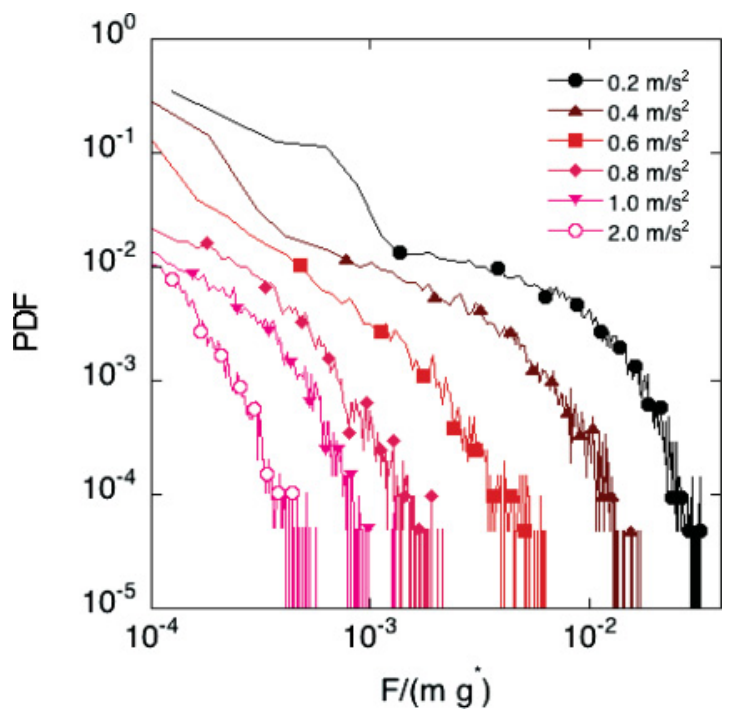

FIG. 6. (Color online) From the simulations, the force at each contact can be calculated. The figure presents the force distribution function for different apparent gravity $g^{\star}$ (see legend) obtained for the centrifuge simulations. 


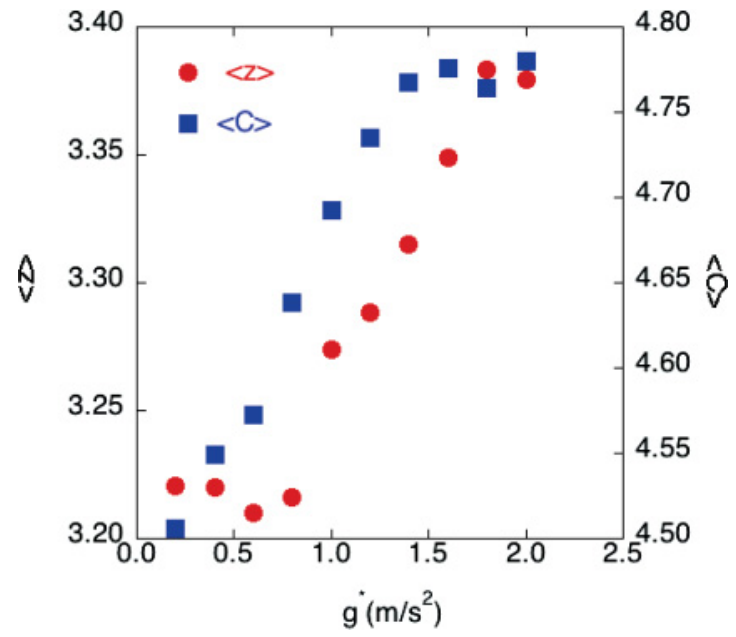

FIG. 7. (Color online) From simulations: Average number of contacts per bead $\langle z\rangle$ (red bullets) as a function of the reduced acceleration. In parallel, the average number of neighbors per bead $\langle C\rangle$ (blue squares) is presented. A neighbor is located at $2.2 R$ from the center of mass of another bead.

The numerical simulation allows us to measure the force distribution in the assembly. In Fig. 6, the probability distribution function (PDF) of the forces acting at the contacts is represented for different accelerations. Note that we consider only the forces between the beads. Indeed, the friction forces between the beads and the plate are not considered to build these probability distribution functions because we want to evidence how the forces are distributed in the plane of the layer. Let us compare two extreme situations at $g^{\star}=0.2$ and $2.0 \mathrm{~m} / \mathrm{s}^{2}$. At $g^{\star}=0.2 \mathrm{~m} / \mathrm{s}^{2}$, the distribution is large. A step is observed at about $10^{-3}$. This is characteristic that two networks exist: (i) a weak force network and (ii) a strong force network that represents the arches. On the other hand, at $g^{\star}=2.0 \mathrm{~m} / \mathrm{s}^{2}$, the distribution is narrow. This is the signature that the contacts between the beads become equivalent and support the same horizontal constraint. The packing is more ordered and consequently denser. The results shown in Fig. 6 suggest a continuous transition between ordered and disordered packing when the apparent gravity is increased.

The average number of contacts per beads $\langle z\rangle$ has been measured from the simulations. Again, we only consider bead-bead contacts. They are plotted (red bullets) as a function of the apparent gravity in Fig. 7. The number of contacts increases with $g^{\star}$. In parallel, the average number of beads located at the vicinity of a bead $\langle C\rangle$ is reported (blue squares). A bead is considered as a neighbor of another grain when its center of mass is located at less than 2.2 times the radius from the center of mass of the considered bead. This value as a function of the apparent gravity also increases and even saturates toward 4.8. This value is quite far from 6 , as is expected for a crystallized phase. This is probably due to frustration and to initial conditions. However, both values $(\langle z\rangle$ and $\langle C\rangle$ ) indicate the ordering of the packing when the apparent gravity is increased.

The visualization of the force network is shown in Fig. 8. The links between contacting grains have been represented. These links are thicker when the force acting on that contact is larger. In so doing, it is possible to reveal the structure of the force network. On the left, the force distribution has been presented for a monolayer of grains formed when the gravity is $0.2 \mathrm{~m} / \mathrm{s}^{2}$, and on the right when the gravity is $1.6 \mathrm{~m} / \mathrm{s}^{2}$. Note that the thickness of a link represents the force relative to the maximum force encountered in the pile; the graphics can be confronted only qualitatively. The force network is found to be more homogeneous in the large gravity case. Indeed, only small arches can be observed in this case. On the other hand, large force chains are found in the low gravity built monolayer. The difference of the monolayer heights is a direct measurement of the volume fraction difference and is a direct consequence of the existence of arches in the low gravity situation.

\section{CONCLUSION}

A horizontal monolayer of noncohesive spherical grains is submitted to an in-plane apparent gravity, either by tilting
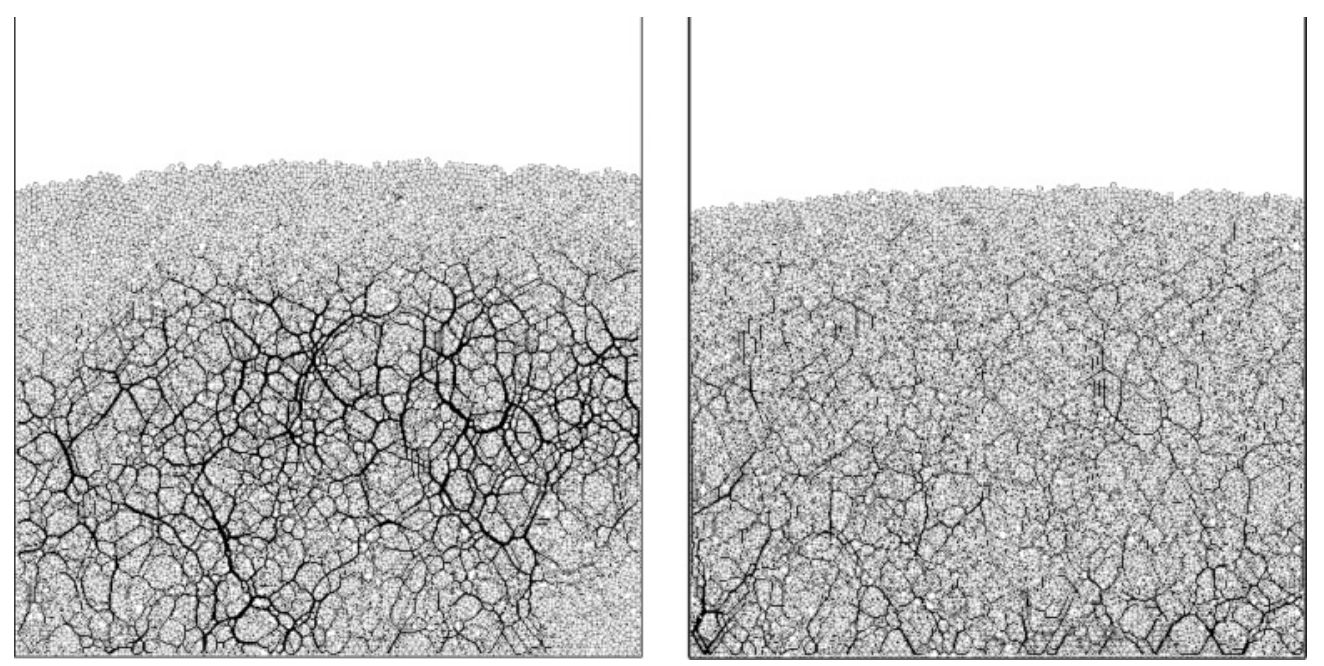

FIG. 8. Force distribution obtained from the simulation in a pile generated under $g^{\star}=0.2 \mathrm{~m} / \mathrm{s}^{2}$ (left) and $g^{\star}=1.6 \mathrm{~m} / \mathrm{s}^{2}$ (centrifuge). The link between two contacting grains is thicker when the force is larger. $N=8000$ in both cases. 
the plate or by a centrifugal force. The packing is generated under the chosen apparent gravity. The volume fraction is then measured. A comparison of both methods has been done. The centrifuge method allows us to conserve a constant normal force. We found that the space occupied by the grains decreases exponentially (saturating) when the apparent gravity is increased between 0 and $1 \mathrm{~m} / \mathrm{s}^{2}$. For large accelerations, the density saturates toward the value of the $2 \mathrm{D}$ random close packing $(\approx 0.9)$.

Considering that the saturated regime is governed by geometrical constraints on the surface distribution of the Voronoi cells, a model describing the evolution of the mean volume fraction with the apparent gravity has been deduced and found to be in good agreement with the observations. The numerical simulations have finally proved that the vol- ume fraction reflects the arching density in the monolayer. At low gravities, the monolayer weight is supported by a small number of force chains; the pile is not ordered and is consequently characterized by a small volume fraction. On the other hand, at high accelerations, the packing is more ordered and the forces better distributed between the grains.

\section{ACKNOWLEDGMENTS}

S.D. is an F.R.S.-FNRS research associate. G.L. is an F.R.S.-FNRS researcher. This work is financially supported by the FEDER/Wallonia Region project called MORECAR and by the INANOMAT project (Grant No. IAP P6/17) of the Belgian Science Policy.
[1] D. J. Scheeres, C. M. Hartzell, P. Sánchez, and M. Swift, Icarus 210, 968 (2010).

[2] J. Duran and A. Reisinger, Sands, Powders, and Grains: An Introduction to the Physics of Granular Materials (SpringerVerlag, New York, 1999).

[3] D. M. Mueth, H. M. Jaeger, and S. R. Nagel, Phys. Rev. E 57, 3164 (1998).

[4] S. Dorbolo, Eur. Phys. J. E 17, 77 (2005).

[5] T. Scheller, C. Huss, G. Lumay, N. Vandewalle, and S. Dorbolo, Phys. Rev. E 74, 031311 (2006).

[6] A. J. Batista-Leyva, F. Pacheco-Vázquez, and J. C. Ruiz-Suárez, Phys. Rev. E 82, 031304 (2010).

[7] F. Pacheco-Vázquez and J. C. Ruiz-Suárez, Nature Commun. 1, 123 (2010).

[8] M. A. Aguirre, J. G. Grande, A. Calvo, L. A. Pugnaloni, and J.-C. Géminard, Phys. Rev. Lett. 104, 238002 (2010).

[9] G. Y. Onoda and E. G. Liniger, Phys. Rev. Lett. 64, 2727 (1990).

[10] N. Isert, C. C. Maa $\beta$, and C. M. Aegerter, Eur. Phys. J. E 28, 205 (2009).
[11] F. Ludewig, S. Dorbolo, T. Gilet, and N. Vandewalle, Europhys. Lett. 84, 44001 (2008).

[12] F. Ludewig, S. Dorbolo, F. Boschini, R. Cloots, and N. Vandewalle, Can. J. Phys. 89, 779 (2011).

[13] G. Lumay and N. Vandewalle, Phys. Rev. Lett. 95, 028002 (2005).

[14] R. B. S. Oakeshott and S. F. Edwards, Physica A 189, 188 (1992).

[15] T. Aste and D. Weaire, The Pursuit of the Perfect Packing (Taylor \& Francis, London, 2000).

[16] G. F. Voronoi, J. Reine Angew. Math. 134, 198 (1908).

[17] T. Aste, J. Phys. Condens. Matter 17, S2361 (2005).

[18] T. Aste, Phys. Rev. Lett. 96, 018002 (2006).

[19] F. Lechenault, F. da Cruz, O. Dauchot, and E. Bertin, J. Stat. Mech. (2006) P07009.

[20] The bead-plate friction was also measured by gluing beads on a slider. The slider was laid on the plate that was inclined until the slider moved. The measurement of the angle allowed us to deduce the friction $\mu_{\mathrm{b}-\mathrm{pl} \text {,exp }}=0.31 \pm 0.03$. 